\title{
Circuit
}

Musiques contemporaines

\section{In Memoriam Pierre Boulez (1925-2016) : l'état de la recherche}

\section{Jonathan Goldman}

Volume 26, numéro 2, 2016

URI : https://id.erudit.org/iderudit/1037305ar

DOI : https://doi.org/10.7202/1037305ar

Aller au sommaire du numéro

Éditeur(s)

Les Presses de l’Université de Montréal

ISSN

1183-1693 (imprimé)

1488-9692 (numérique)

Découvrir la revue

Citer ce document

Goldman, J. (2016). In Memoriam Pierre Boulez (1925-2016) : l'état de la recherche. Circuit, 26(2), 73-76. https://doi.org/10.7202/1037305ar d'utilisation que vous pouvez consulter en ligne.

https://apropos.erudit.org/fr/usagers/politique-dutilisation/ 


\section{ACTUALITÉS}

\section{In Memoriam Pierre Boulez (1925-2016) : l'état de la recherche ${ }^{1}$}

Jonathan Goldman

Avec la disparition de Pierre Boulez, une impression immanquable de la fin d'une époque nous empare, celle des temps «héroïques» du modernisme musical de l'après-guerre et de ses enjeux esthétiques et politiques. Avec le décès - attendu mais non moins navrant - du dernier avatar de la génération européenne de l'immédiat après-guerre, nous arrivons au terme d'une triste chaîne dont les maillons sont constitués de compositeurs contemporains influents disparus avant lui (Nono en 1990, Cage en 1992, Goeyvaerts en 1993, Xenakis en 2001, Berio en 2003, Ligeti en 2006, Stockhausen en 2007, Pousseur en 2009, pour ne nommer que ceux-là), sans parler du Britannique Peter Maxwell-Davies, mort deux mois après lui. Inévitables formules de la part des journalistes de publications généralistes qui mettent "un point véritablement final au $\mathrm{xx}^{\mathrm{e}}$ siècle musical avant-gardiste qu'il avait notablement contribué à façonner ${ }^{2}$ », car les phases artistiques successives de Boulez se miroitaient dans celles de toute une génération, décennie après décennie, qui allaient d'un post-webernianisme «paramétrique » à des expériences électroacoustiques, des œuvres «mobiles» aléatoires au traitement électronique en temps réel, de l'art de la miniature néoexpressionniste à la récupération de la grande forme. Boulez a également représenté l'avant-garde musicale depuis 1945 aux yeux d'un large public, grâce à son métier de chef d'orchestre (New York Philharmonic, ввс Symphony Orchestra, Cleveland, entre bien d'autres orchestres prestigieux). N'oublions pas que Pierre Boulez a aussi eu un impact significatif sur le développement d'institutions musicales, surtout en France, si on pense à l'Ircam, à l'Ensemble intercontemporain, à la Cité de la musique, jusqu'à l'ouverture récente de la Philharmonie de Paris. L'histoire de la revue Circuit rencontre aussi la figure de Boulez à plusieurs moments significatifs de son histoire: rappelons que le premier rédacteur en chef et cofondateur de la revue, Jean-Jacques Nattiez, est l'éditeur, depuis le début des années 1980, des écrits de Pierre Boulez (la série «Points de repère»), et a longtemps codirigé une collection de livres avec le compositeur français chez Christian Bourgois éditeur. Circuit a aussi consacré un numéro entier à la tournée qu’a fait Boulez au Canada en mai-juin 1991, dans le vol. 3, nº 1 (1992) («Boulez au Canada: portrait d'impact»).

Il est quelque peu consolant de penser que la dernière année de l'existence de Boulez a été remplie d'activités musicales qui célébraient l'œuvre du compositeur, à l'occasion de ses 90 ans. Toutefois, en raison du faible état de sa santé, Boulez n’a pas pu assister à la quasi-totalité de ces activités, mais a dû néanmoins 


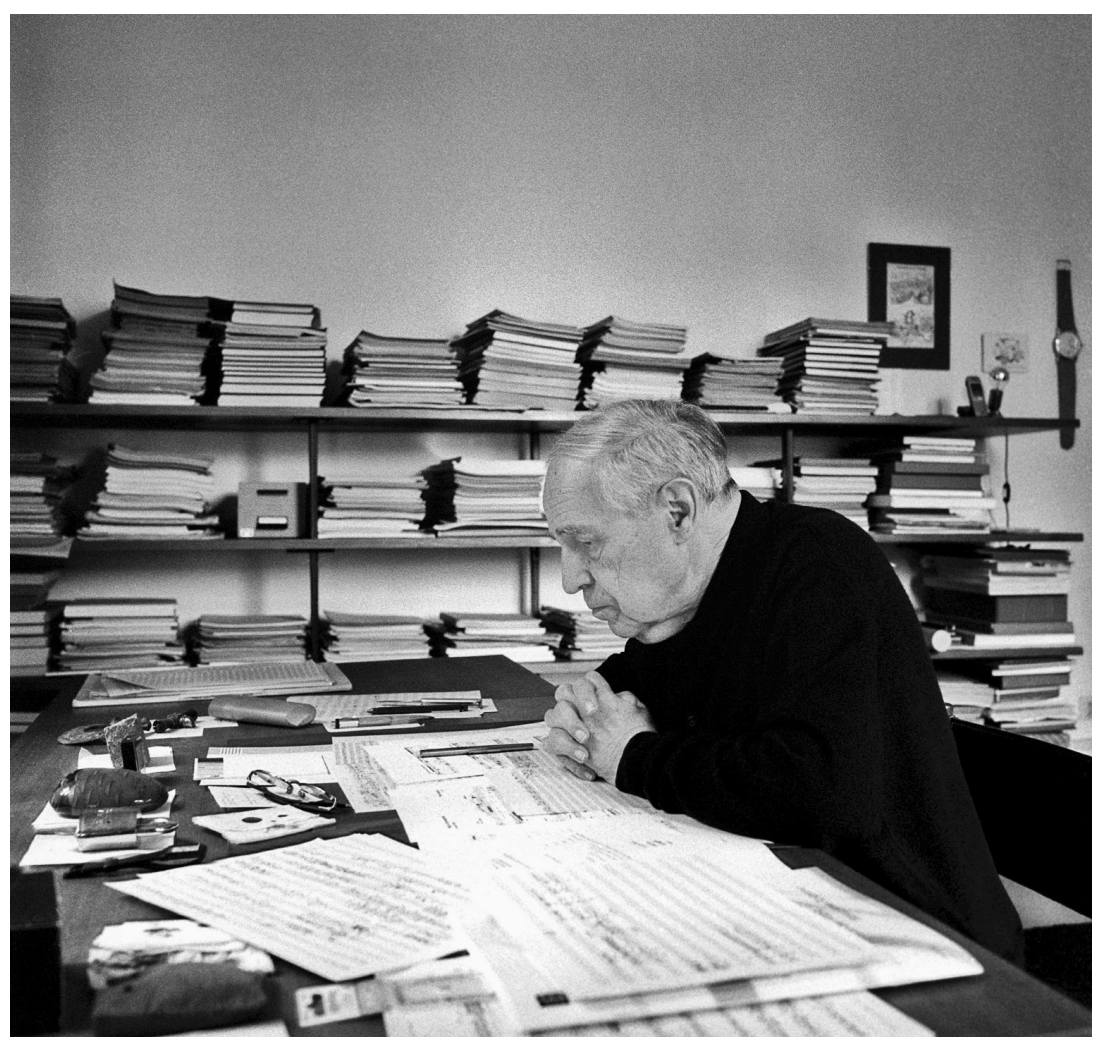

être mis au courant de toute l'énergie déployée à travers le monde pour rendre hommage au compositeur, chef d'orchestre et intellectuel d'exception qu'il était. Au cours de l'année 2015, les deux orchestres étatsuniens les plus étroitement liés au chef Boulez, ceux de Cleveland et de Chicago, ont célébré sa musique dans le cadre de concerts majeurs, l'orchestre de Chicago ayant même réalisé un film-concert multimédia en collaboration avec l'architecte Frank Gehry³. À Paris, une exposition sur la vie et l'œuvre de Pierre Boulez fut présentée au Musée de la musique et à la Philharmonie de Paris. À Londres, l'orchestre de la BBC s'est associé au London Symphony Orchestra et à l'Ensemble intercontemporain pour proposer un «Boulez disparition du compositeur le 5 janvier 2016, d'autres orchestres et ensembles ont proposé des hommages à Boulez en ajoutant telle ou telle de ses œuvres au programme de leurs concerts. Quant aux enregistrements disponibles de ses œuvres, déjà deux ans avant l'année de son $90^{\circ}$ anniversaire, Deutsche Grammophon avait édité un coffret de 13 disques compacts proposant les «Complete Works» de Boulez, annonçant ainsi à même le titre qu'aucune autre œuvre n'allait s'ajouter au catalogue des opus du compositeur, malgré les rumeurs récentes selon lesquelles Boulez aurait entamé un projet d'opéra à la Scala qui s'ajouterait au projet d'achèvement de plusieurs œuvres restées inabouties (la Troisième sonate, le Livre pour quatuor, Répons, etc.) - aux dires du musicien. Désormais nous 
devrons nous habituer au caractère achevé-quoiqueinachevé de bon nombre d'œuvres de ce compositeur profondément marqué par l'esthétique joycéenne du «work-in-progress» et du «Livre» mallarméen.

Or, le rôle du musicologue, tel que je le conçois, n'est pas de chanter des éloges, ni de distribuer des palmarès, ni de couronner les futurs élus du Panthéon'. Le musicologue est d'abord un chercheur, et le meilleur hommage qu'il peut faire à Pierre Boulez consiste en des recherches toujours plus poussées sur ses projets artistiques, culturels et institutionnels. Heureusement, les dix dernières années ont vu une faste récolte de travaux scientifiques sur Boulez. De nouvelles approches ont été proposées, entre autres par Catherine Steinegger, auteure d'un ouvrage sur la production boulézienne pour la scène (Pierre Boulez et le théâtre, Bruxelles, Mardaga, 2012), ou Edward Campbell, qui explore les fondements philosophiques, à la frontière du structuralisme et du poststructuralisme, de la pensée de Boulez (Boulez, Music and Philosophy, Cambridge University Press, 2010). Les dernières années ont également vu des avancées significatives sur le plan analytique par des music theorists tels que Catherine Losada et Ciro Scotto, et surtout, un gain en études de type génétique (sketch studies), rendues possibles grâce à la présence depuis 1986 du Fonds Boulez au sein de la Fondation Paul Sacher à Bâle, par des chercheurs tels que Robert Piencikowski, Pascal Decroupet, Peter O'Hagan, Paolo Dal Molin ou Brice Tissier. La Fondation Sacher a par ailleurs édité deux magnifiques (et surdimensionnées) éditions en fac-similé de manuscrits d'œuvres de Boulez (Le marteau sans maître ${ }^{6}$ et «Tombeau », dernier mouvement de Pli selon pli ${ }^{7}$ ). Ces documents viennent s'ajouter à un corpus déjà passablement vaste de travaux sur le compositeur français, si on pense qu'une bibliographie des articles sur Boulez publiée dans une revue de musicologie comporte 16 pages - et ce, même si elle a été publiée il y a 44 ans $^{8}$ ! Depuis ce temps, le nombre de travaux, y compris des articles, des thèses et des livres, ne cesse de croître9. Pourtant, vu sous un autre angle, il est possible d'avoir l'impression que la recherche sur Boulez et sur ses contemporains ne fait que commencer aujourd'hui, à l'heure où l'influence écrasante des récits modernistes sur l'historiographie de la musique occidentale se tempère. Cette nouvelle situation permet aux chercheurs de ne plus rester ensorcelés par le discours hyper confiant des compositeurs eux-mêmes, un discours toujours infusé de matière première historiographique, prêt à servir, pour ainsi dire, aux musicologues, mais à leurs risques et périls. C'est une situation qui a été récemment explorée par Charles Wilson et Anne-Sylvie Barthel-Calvet ${ }^{10}$, et qui, dans un numéro de Circuit que j’ai codirigé avec mon collègue Nicolas Donin, avait déjà été abordée lorsque nous nous sommes posé la question suivante :

[C]omment parler aujourd'hui de la haute modernité produite et représentée par Darmstadt, sans reconduire ni la téléologie fiévreusement pratiquée par les acteurs de cette histoire ni la grisaille bienveillante d'une musicologie historique prompte à la digestion académique de la «musique contemporaine $»^{11}[?]$

En effet, des travaux relativement récents ont pu proposer des intrigues historiographiques qui divergent considérablement de celles proposées par les compositeurs eux-mêmes dont Boulez, comme l'étude sociologique désormais classique de Georgina Born sur l'Ircam², et celle de Mark Carroll sur les Structures pour deux pianos de Boulez dans le contexte de la Guerre froide et de l'événement «L'Euvre du Xxe siècle» organisé à Paris en 1954 par Nicolas Nabokov ${ }^{13}$. Dans ma propre recherche récente, je me penche sur les rapports qu'ont entretenus des compositeurs comme Boulez avec la technologie de reproduction du son. Une première étude, qui porte sur la première œuvre proprement orchestrale de Boulez, Doubles (1958), sera publiée cette année dans un recueil consacré à Boulez ${ }^{14}$. Labondance de ces travaux récents démontre, s'il en était besoin, 
qu'il reste beaucoup de choses à dire et à apprendre sur les tendances musicales représentées par une figure titanesque comme Pierre Boulez. Et de nouvelles façons d'entendre ses œuvres également. De quoi confirmer notre impression que sa musique reste fascinante, chatouillante, dérangeante, pertinente.

1. [NDLR] Une première version de cet article est parue en anglais sur le blogue Musicology Now de l'American Musicological Society: $<$ http://musicologynow.ams-net.org/2015/08/boulez-turns-90scholarship-on-boulez.html> (consulté le 28 avril 2016).

2. Renaud Machart (2016), "Mort de Pierre Boulez, symbole d'un xxe siècle musical avant-gardiste", Le Monde (6 janvier), $<$ www.lemonde.fr/disparitions/article/2016/01/06/mort-ducompositeur-et-chef-d-orchestre-pierre-boulez_4842501_3382.html> (consulté le 28 avril 2016).

3. L'événement a eu lieu le 14 novembre 2014; voir:

$<$ http://csosoundsandstories.org/beyond-the-score-a-pierre-dream> (consulté le 28 avril 2016).

4. À propos de cette journée, voir: < www.barbican.org.uk/music/ event-detail.asp?!ID=15978> (consulté le 28 avril 2016).

5. Un rôle que plusieurs n'ont pas hésité à jouer à l'époque des débuts de la carrière de Boulez. Voir par exemple, Pierre Souvtchinsky
(1954), "À propos d'un retard", Cahiers de la Compagnie Madeleine Renaud-Jean-Louis Barrault, $2^{\mathrm{e}}$ année, $\mathrm{n}^{\circ}$ 3, Paris, Julliard, p. 127.

6. Pascal Decroupet (dir.) (2005), Le marteau sans maître: fac-similé de l'épure et de la première mise au net de la partition, Veroffentlichungen der Paul Sacher Stiftung, London, Schott.

7. Robert Piencikowski (dir.) (2010), Tombeau: fac-similés de l'épure et de la première mise au net de la partition, Vienna-London-New York, Universal Edition.

8. Michael Fink (1972), "Pierre Boulez: A Selective Bibliography", Current Musicology, no 13, p. 135-150.

9. En janvier 2016, j’ai publié une bibliographie annotée sélective sur Boulez sur la plateforme Oxford Bibliographies,

<www.oxfordbibliographies.com> (consulté le 28 avril 2016).

10. Charles Wilson (2004), "György Ligeti and the Rhetoric of Autonomy", Twentieth-Century Music, vol. 1, n ${ }^{\circ}$ 1, p. 5-28, et AnneSylvie Barthel-Calvet (dir.) (2011), Propositions pour une historiographie critique de la création musicale après 1945, Metz, Centre de recherche universitaire lorrain d'histoire.

11. Nicolas Donin, "Les cours d'été de Darmstadt, du mythe au chantier" (2005), Circuit, musiques contemporaines, vol. $15, n^{\circ} 3$, p. 8.

12. Georgina Born (1995), Rationalizing Culture, Berkeley, University of California Press.

13. Mark Carroll (2006), Music and Ideology in Cold War Europe, Cambridge, Cambridge University Press.

14. Edward Campbell et Peter O'Hagan (dir.) (2016), Boulez Studies, Cambridge, Cambridge University Press. 